Published in final edited form as:

Sports Med. 2017 May ; 47(5): 1011-1019. doi:10.1007/s40279-016-0618-6.

\title{
Association between Contact Sports and Colonization with Staphylococcus aureus in a Prospective Cohort of Collegiate Athletes
}

\author{
Natalia Jiménez-Truque, PhD, MSCI ${ }^{1}$, Elizabeth J. Saye, BS ${ }^{1}$, Nicole Soper, MT $^{1}$, Benjamin \\ R. Saville, $\mathrm{PhD}^{2}$, Isaac Thomsen, MD, MSCI ${ }^{1}$, Kathryn M. Edwards, MD'1, and C. Buddy \\ Creech, MD, MPH ${ }^{1}$ \\ ${ }^{1}$ Division of Pediatric Infectious Diseases, Vanderbilt University Medical Center, Nashville, \\ Tennessee, USA \\ ${ }^{2}$ Department of Biostatistics, Vanderbilt University School of Medicine, Nashville, Tennessee, \\ USA
}

\begin{abstract}
Objective-Athletes have higher risk of infection with Staphylococcus aureus than the general population. Most studies in athletes have included primarily male contact sports participants and have not assessed $S$. aureus carriage over time. We aimed to examine the epidemiology and risk factors of $S$. aureus carriage in a cohort of male and female collegiate athletes.
\end{abstract}

Study Design-We conducted a prospective cohort study of 377 varsity collegiate athletes from August 2008 to April 2010. A baseline questionnaire ascertained risk factors for colonization. Nasal and oropharyngeal swabs were obtained at enrollment and monthly thereafter to detect $S$. aureus colonization. The primary outcome was $S$. aureus colonization, both with methicillinsusceptible and methicillin-resistant $S$. aureus, as defined by bacterial culture and molecular confirmation. Secondary outcomes were time to colonization with $S$. aureus and carriage profile, defined as non-carrier, intermittent carrier or persistent carrier.

Results-Overall, 224 contact sports and 153 non-contact sports athletes were enrolled. Contact sports athletes had higher risk of carrying $S$. aureus over time: they had higher odds of being colonized with MRSA (OR, 2.36; 95\% CI, 1.13-4.93) and they tended to carry $S$. aureus for longer periods of time (intermittent carriage OR, 3.60; 95\% CI, 2.02-6.40; persistent carriage OR, 2.39;

Corresponding Author: Natalia Jiménez-Truque, PhD, MSCI, Research Instructor, Pediatric Infectious Diseases, Vanderbilt University Medical Center, 1161 21st Avenue South, D-7215 MCN Nashville, Tennessee 37232, Phone: 615-322-3076,

natalia.jimenez@vanderbilt.edu.

Financial Disclosure: All authors have no financial relationships relevant to this article to disclose.

Disclosure of potential conflicts of interest: Conflict of Interest: $\mathrm{CBC}$ has received research grants from Pfizer, Inc. for S. aureus vaccine research; Novartis Vaccines for S. aureus immune response studies; and Diatherix Laboratories for S. aureus diagnostic assays. Authors NJT, EJS, NS, BRS, IPT and KME declare that they have no conflict of interest.

NJT had full access to all the data in the study and takes responsibility for the integrity of the data and the accuracy of the data analysis.

Compliance with Ethical Standards: Research involving Human Participants: Ethical Approval: All procedures performed in studies involving human participants were in accordance with the ethical standards of the institutional and/or national research committee and with the 1964 Helsinki declaration and its later amendments or comparable ethical standards.

Informed consent: Informed consent was obtained from all individual participants included in the study. 
95\% CI, 1.21-4.72). Athletes engaged in contacts sports also acquired $S$. aureus more quickly (HR, $1.61 ; 95 \%$ CI, 1.02-2.55).

Conclusions-S. aureus carriage was common in contact sports athletes, particularly football. These findings suggest that efforts to prevent transmission of $S$. aureus among athletes should be focused on contact sports teams.

\section{Keywords}

Staphylococcus aureus; athletes; colonization; epidemiology; sports

\section{Introduction}

There are more than 400,000 collegiate athletes in the US,[1] and these athletes are at increased risk of Staphylococcus aureus infections, most commonly skin and soft tissue infections (SSTIs). Community-associated methicillin-resistant $S$. aureus (CA-MRSA) has become the most common bacterial pathogen in the US and often leads to recurrent skin and soft tissue infections (SSTI) in otherwise healthy individuals.[2] One risk factor for $S$. aureus infection is asymptomatic colonization - approximately $30 \%$ of the general population are colonized with $S$. aureus, most notably in the anterior nares.[3-6] Other body sites may also harbor $S$. aureus, $[7,8]$ though the characteristics of colonization in these sites, such as the oropharynx, axilla, groin and skin, have not been completely defined. Among those that are colonized, some groups are at high risk for disease, such as prisoners, $[9,10]$ military recruits,[6] and athletes,[11-13] and are ideal populations in which to study the longitudinal characteristics of $S$. aureus colonization and disease.

Although knowledge of $S$. aureus colonization has increased, the biology of nasal colonization remains incompletely understood and it is not known why some colonized individuals do not develop disease. Most studies of at-risk populations, including athletes, have focused on nasal colonization only during outbreaks [12] and have focused primarily on contact sports participants.[14, 15] Additionally, most colonization studies have not distinguished between non-carriers, intermittent carriers, and persistent carriers, who differ in both the frequency of colonization and subsequent risk of disease.[5, 16, 17] Without a broader understanding of $S$. aureus colonization, strategies for management and prevention of CA-MRSA in sports will remain unclear.

In this two-year prospective cohort of varsity collegiate athletes, we assessed $S$. aureus colonization in order to compare the dynamics of nasal and oropharyngeal staphylococcal colonization over time among contact and non-contact sports athletes. The primary hypothesis of this study was that athletes in contact and non-contact sports would differ in their patterns of $S$. aureus colonization.

\section{Patients and Methods \\ Population}

From August 2008 to April 2010, all varsity athletes and athletic trainers at Vanderbilt University were invited to participate in the study. The study was approved by the Vanderbilt 
Human Subjects Protection Program and all participants provided written informed consent prior to any study-related procedures. Athletes were categorized into either contact or noncontact sports, as defined by the American Academy of Pediatrics (AAP),[18] with the exception of baseball players (defined by AAP as 'limited-contact') and athletic trainers, who were both classified as non-contact athletes. No athletes switched sports during the study. A baseline questionnaire ascertained risk factors for colonization.

\section{Specimen Collection and Microbiology Procedures}

Nasal and oropharyngeal samples were obtained at enrollment and monthly thereafter until the end of the study. All samples were obtained and processed as previously described.[19, 20] Coagulase positive isolates recovered from mannitol salt agar plates containing $6 \mu \mathrm{g} / \mathrm{mL}$ of oxacillin were considered potential MRSA isolates. Bacterial DNA from these isolates was extracted and purified (MoBio Laboratories, Inc., Carlsbad, CA), and the presence of the $m e c A$ gene (indicative of MRSA) was confirmed by polymerase chain reaction (PCR) using previously described methods.[21]

\section{Molecular Characteristics of MRSA Isolates}

Staphylococcal Cassette Chromosome mec (SCCmec) type and presence of PantonValentine Leukocidin (PVL) genes were determined using previously described methods. [22-24] Repetitive-element, sequence-based PCR (rep-PCR, DiversiLab System, bioMerieux, Inc) was used to determine overall genetic relatedness of isolates.

\section{Statistical Analysis}

Data were recorded in the Vanderbilt Research Data Capture System (REDCap).[25] Univariate analysis of colonization, demographics and medical history was performed using Pearson's chi-squared or Fisher's exact test for categorical characteristics, as appropriate. Since monthly samples were not always collected on all athletes, the exact date when an athlete became colonized might not have been known; thus, some data were intervalcensored. Further, those athletes who remained not colonized by the end of the study were right-censored. Given the presence of interval-censored data, the usual semi-parametric Cox proportional hazards model could not be used. Instead, a parametric survival analysis with a Weibull distribution, accounting for interval- and right-censored data, was performed to compare hazard ratios for becoming colonized with any $S$. aureus in contact vs. non-contact sports' athletes; subjects colonized at baseline were excluded from this analysis. The analysis was repeated to include only freshmen. For assessing the association between type of sport and colonization status over time, and to evaluate the association between persistent $S$. aureus carriage and colonization site, multinomial mixed models were used to account for the correlation between the repeated measures within the same individual. For the model that assessed the association between sport and colonization, the outcome categories were no colonization (reference), colonization with MSSA, or colonization with MRSA. For the model that assessed the association between persistent colonization and site, the outcome was categorized as nasal colonization only (reference), oropharyngeal only, or colonization in both sites. For all mixed models, time (months) was included as a linear term; given the limited number of predictors allowed in each model, we felt that the included variables were more important factors to consider than time. The only time-dependent covariate that was 
measured was college year. All other covariates of interest were not time-dependent (e.g. gender, race). A multinomial logistic regression model was used to assess the association between being persistent, intermittent, or non-carriers and playing contact sports; those who were staphylococcal carriers during $\$ 80 \%$ of the times at which they are sampled were classified as persistent carriers; those who were never colonized were non-carriers, and those colonized at least once, but $<80 \%$ of the times at which they were swabbed, were classified as intermittent carriers.[3, 5, 26] To assess whether specific strain-types of MRSA were more likely to cause persistent staphylococcal carriage, a logistic regression analysis was performed; subjects who were non-carriers were excluded from this analysis. All confounders were selected a priori based on prior knowledge and based on the number of predictors that could be reliably entered into each model, given the number of events, to avoid the risk of overfitting these models; there was no dropping or including covariates based on other analyses. Such approaches have been shown in the literature to produce misleading results.[27] Two sensitivity analyses were performed for all models to assess the effect of incomplete follow-up; one adjusted for the number of months subjects were swabbed, categorized as $5,>5$ and $<13$, and $\geq 13$ months (using indicator variables) and the second excluded subjects swabbed $\mathbf{S}$ months. Analyses were conducted using Stata 11.2 (StataCorp LP), SAS 9.3 (SAS Institute Inc.) or R 2.14.2.

\section{Results}

Characteristics of enrolled subjects are presented in Table 1. Of the 377 subjects in the cohort, 224 played contact sports and 153 played non-contact sports. The majority of the athletes were male (57.3\%) and Caucasian (74.3\%). At baseline, 294 (85\%) athletes were enrolled: $72 \%$ from non-contact sports, and $96 \%$ from contact sports. Additional freshmen athletes were enrolled during the second year of the study. A total of 3,291 nasal and oropharyngeal samples were obtained from 377 subjects over 18 sampling periods; 950 from non-contact and 2,341 from contact sports.

\section{Time to colonization with S. aureus}

To assess the time required for athletes not colonized at baseline to become colonized with $S$. aureus, we compared contact and non-contact sports athletes in a time-to-event analysis. At enrollment, 191 subjects (50.66\%) were colonized and excluded from the time-to-event analysis; 186 individuals were included. Included and excluded subjects differed in the proportions of contact sports participants $(51.1 \%$ vs. $67.5 \%$, respectively; $\mathrm{p}=0.001)$, males ( $49.5 \%$ vs. $64.9 \%$, respectively, $\mathrm{p}=0.002$ ) and persistent carriers ( $0 \%$ vs. $45.5 \% ; \mathrm{p}<0.001$ ); all other characteristics did not differ significantly between the groups. Of these 186 subjects, 56 became colonized with MSSA and 40 with MRSA. The median time to colonization with $S$. aureus was 10 months. For contact sports participants, the median time to colonization was seven months, and for those in non-contact sports, 17 months (log-rank test $\mathrm{p}=0.03$; Figure 1). Based on an adjusted survival analysis, the hazard of colonization with $S$. aureus for athletes in contact sports was $61 \%$ higher than for athletes in non-contact sports (hazard ratio [HR], 1.61; 95\% CI, 1.02-2.55; Table 2). Other variables included in the model were not statistically significant. When looking at the Kaplan-Meier curve for the time to becoming colonized with $S$. aureus by type of sport, the curves had similar shapes 
and the separation between them was fairly constant across time, indicating the proportional hazards assumption was met (Figure 1).

To assess whether new team members also exhibited more rapid time to colonization in contact sports vs. non-contact sports, we restricted the previous analysis to freshmen. Of 79 subjects included in this analysis, 21 became colonized with MSSA and 19 with MRSA. The median time to colonization in freshmen was also 10 months. For freshmen participating in contact sports, the median time to colonization was six months, and for those in non-contact sports, $>20$ months (log-rank test $\mathrm{p}=0.03$ ). The hazard of becoming colonized with $S$. aureus for freshmen in contact sports was $90 \%$ higher than for freshmen in non-contact sports (HR, 1.89; 95\% CI, 1.04-3.44; Table 2).

\section{Colonization and Sport}

To assess risk factors associated with $S$. aureus colonization, demographics and medical history were linked to each subject's colonization over time. Based on an unadjusted multinomial mixed model, contact sports athletes had higher odds of being colonized with MRSA and MSSA than athletes in non-contact sports $\left(\mathrm{OR}_{\mathrm{MRSA}}, 2.25\right.$; $95 \% \mathrm{CI}, 1.14-4.43$; $\mathrm{OR}_{\text {MSSA }}, 1.68 ; 95 \% \mathrm{CI}, 1.06-2.65$, Table 3 ). After adjusting for confounders, contact sports participants had significantly higher odds of being colonized with MRSA than not being colonized when compared with athletes in non-contact sports (OR, 2.36; 95\% CI, 1.13-4.93; Table 3). We also observed a trend towards contact sports participants having higher odds of MSSA colonization (OR, 1.57; 95\% CI, 0.94-2.62). A history of $S$. aureus infection was associated with increased odds of MRSA carriage in this cohort (OR, 5.35; 95\% CI, 1.51-18.87). Other associations that were assessed are shown in Table 3. The multiplicative interaction between contact sport and gender was not statistically significant (data not shown).

\section{Persistent S. aureus Colonization and Sport}

The observed prevalence of each carrier profile is shown in Table 1. To assess whether the frequency of persistent, intermittent and non-carriers of $S$. aureus differed by the type of sport in which athletes participated, we categorized subjects into the three carrier profiles and performed a multinomial logistic regression model. Sixty-one percent of the individuals who were non-carriers belonged to non-contact sports teams, while $68 \%$ of intermittent carriers and $60 \%$ of persistent carriers belonged to contact sports teams (Figure 2). Males comprised the majority of intermittent and persistent carriers, while women were the majority among non-carriers (data not shown).

Based on a multinomial logistic regression model, athletes in contact sports were three times more likely to be intermittently colonized and twice as likely to be persistently colonized, than non-contact sports athletes $\left(\mathrm{OR}_{\text {Intermittent }}, 3.42\right.$; 95\% CI, 2.03-5.74; OR $\mathrm{Persistent}_{\text {, }}$.33; 95\% CI, 1.28-4.27). The observed associations remained statistically significant after adjusting for gender, race and college year $\left(\mathrm{OR}_{\text {Intermittent }}, 3.60 ; 95 \% \mathrm{CI}, 2.02-6.40\right.$; $\left.\mathrm{OR}_{\text {Persistent }}, 2.39 ; 95 \% \mathrm{CI}, 1.21-4.72\right)$. These results indicate that athletes in contact sports teams not only have higher prevalence of $S$. aureus colonization, but are also colonized for longer periods of time than athletes in non-contact sports teams. 


\section{Persistent Colonization and SCCmec type of Colonization Isolates}

To examine if MRSA isolates with particular characteristics were more likely to cause persistent colonization, we linked the carrier profile with the type of colonizing MRSA isolate and performed a logistic regression model; athletes who were non-carriers were excluded from this analysis. The percentage of athletes with at least one MRSA isolate with SCCmec type IV was significantly higher among persistent $S$. aureus carriers than among intermittent carriers $(67$ (77.01\%) vs. 85 (42.50\%), respectively; chi-squared $\mathrm{p}<0.001)$. The six athletes who carried PVL-encoding MRSA isolates were intermittent carriers; thus, PVL predicted the carrier profile perfectly and was not included in the model. Based on a logistic regression model, adjusting for sport, gender, and race, athletes who carried at least one MRSA isolate with SCCmec type IV had significantly higher odds of being persistent carriers rather than intermittent carriers (unadjusted OR, 4.53; 95\% CI, 2.55-8.04; adjusted OR, 5.01; 95\% CI, 2.75-9.12).

\section{Persistent Colonization and Colonization Site}

To examine whether persistent $S$. aureus carriers were colonized preferentially in different body sites, the site of colonization over time was linked to the carrier profile, using a multinomial mixed model; athletes who were non-carriers were excluded from this analysis. The odds of oropharyngeal carriage alone were significantly lower than for nasal carriage alone for persistent carriers, as compared with intermittent carriers, even after adjusting for sport, gender, race, and college year (unadjusted: OR, 0.16; 95\% CI, 0.08-0.31; adjusted OR, 0.16; 95\% CI, 0.08-0.31). Conversely, persistent carriers were more likely than intermittent carriers to have both nasal and oropharyngeal colonization than to have nasal colonization alone (unadjusted OR, 2.05; 95\% CI, 1.40-3.02; adjusted OR, 2.05; 95\% CI, 1.40-3.02). These results suggest that carrier profiles might differ not only in the duration of $S$. aureus colonization but may also differ in the body sites that harbor $S$. aureus.

\section{Genetic Relatedness among MRSA Colonization Isolates}

To assess whether the types of MRSA that colonize contact sports athletes differ from those that colonize non-contact sports athletes, we compared the frequency of SCCmec IV, PVL+ isolates in both groups of athletes. Among non-contact athletes, 42 (27.35\%\%) had 455 SCCmec IV MRSA isolates during the study, and 110 (49.11\%) contact sports athletes had 148 such isolates (chi-squared $\mathrm{p}<0.01$ ). Three contact athletes had $18 \mathrm{PVL}+$ isolates, and three non-contact athletes had 12 such isolates. Colonization isolates were highly heterogeneous, and no single genetic lineage predominated.

\section{Discussion}

In this study, we demonstrated that athletes in contact sports become colonized with $S$. aureus more quickly and have higher odds of being persistent $S$. aureus carriers than noncontact sports participants. The frequency of $S$. aureus colonization was high in this cohort; however, $S$. aureus disease was infrequent, with only nine abscesses identified in 7 individuals; two abscesses grew MSSA and seven MRSA (previously published data).[20] These findings have implications for S. aureus colonization surveillance in student athletes, determination of periods of transmission risk, and employment of preventive strategies. 
First, athletes in contact sports became colonized with $S$. aureus more rapidly than those in non-contact sports. While this may be intuitive given the frequency of skin-to-skin contact and mild skin abrasions,[28] this is the first study to demonstrate this difference between types of sport. Moreover, contact sports athletes were more likely to be colonized with MRSA than those in non-contact sports. Other risk factors associated with $S$. aureus colonization were male gender, Caucasian race, and a personal history of $S$. aureus infection, each of which are known risk factors for $S$. aureus disease.[3, 26, 29-32]. Although gender was associated with colonization, this association was independent of the type of sport the athletes played; i.e., there was no gender-sport interaction.

We also observed a higher percentage of intermittent and persistent $S$. aureus carriers among contact sports participants, and a higher percentage of non-carriers among non-contact sports athletes. While in prior studies weekly swabs have often been performed to characterize S. aureus carrier profiles, $[5,16]$ we obtained swabs monthly but for up to 18 months to assess the dynamics of colonization over time in our population. Our sampling periods are consistent with those of van Belkum et al who assessed carrier profiles by obtaining nasal samples every 3-4 weeks.[17] Though there is a potential for misclassification of the carrier profile, the prospective nature of the study makes it likely to be non-differential, and the distribution of carrier profiles is largely consistent with other studies, which have found that approximately $20 \%$ of subjects are persistent carriers, approximately $60 \%$ are intermittent, and approximately $20 \%$ are non-carriers.[3, 4, 26, 33]

Compared with intermittent carriers, persistent carriers had higher odds of being colonized with SCCmec type IV MRSA than with other SCCmec types and were more likely to have both nasal and oropharyngeal carriage. These results suggest that carrier profiles not only differ in their duration of $S$. aureus colonization, but also in the body sites that harbor $S$. aureus and, potentially, in the type of $S$. aureus isolates with which they are colonized. Whether these associations remain true in other populations would require prospective studies in other groups.

Despite being a prospective study in a population with high prevalence of staphylococcal colonization, there are important limitations. Since medical history was assessed through a baseline questionnaire, there is potential for recall bias. Second, misclassification of contact and non-contact sports participation could have occurred. The American Academy of Pediatrics (AAP) classifies sports as contact, limited-contact and non-contact sports;[18] given that only baseball is classified by the AAP as limited-contact, baseball was classified as a non-contact sport for this study. A sensitivity analysis classifying baseball as a contact instead of non-contact sport (Online Table 1 and Online Table 2, Online Material) revealed a stronger association between contact sports and colonization with MRSA over time, suggesting that our original results were more conservative. Third, since some athletes were noncompliant with monthly swabbing, several individuals were absent during any given month's sampling, thus generating missing data. Using mixed models for assessing longitudinal colonization, however, makes the best use of the available data, without discarding subjects with incomplete data. Sensitivity analyses controlling for duration of follow-up or excluding subjects with five or less observations did not change the results 
appreciably for any of the models we performed (Online Table 1 and Online Table 2, Online Material, and other data not shown).

\section{Conclusion}

Contact sports athletes were more likely to be colonized with $S$. aureus than non-contact sports athletes. $S$. aureus colonization occurred more frequently in contact sports athletes and led to a higher prevalence of persistent colonization. These results suggest that prevention efforts aimed at reducing $S$. aureus colonization, and thus potentially reducing infections, should first target contact sports athletes. Where possible, ascertainment of straintype may be beneficial in informing specific interventions, and future studies should focus on identifying the most cost-effective prevention strategies in this high-risk group.

\section{Supplementary Material}

Refer to Web version on PubMed Central for supplementary material.

\section{Acknowledgments}

The authors recognize the nurses, students, trainers, sports medicine physicians, and team coaches that made this work possible.

Funding Source: This work was supported by the Division of Microbiology and Infectious Diseases from the National Institute of Allergy and Infectious Diseases at the National Institutes of Health [K23-AI074830] to CBC; the National Center for Research Resources [UL1 RR024975-01], and is now at the National Center for Advancing Translational Sciences [UL1 TR000445-06]. The content is solely the responsibility of the authors and does not necessarily represent the official views of the NIH.

\section{References}

1. [cited 2013 April 29] NCAA College Athletics Statistics. 2012 May 8. 2012Available from: http:// www.statisticbrain.com/ncaa-college-athletics-statistics/

2. David MZ, Daum RS. Community-associated methicillin-resistant Staphylococcus aureus: epidemiology and clinical consequences of an emerging epidemic. Clin Microbiol Rev. 2010; 23(3): 616-87. [PubMed: 20610826]

3. Kluytmans J, van Belkum A, Verbrugh H. Nasal carriage of Staphylococcus aureus: epidemiology, underlying mechanisms, and associated risks. Clin Microbiol Rev. 1997; 10(3):505-20. [PubMed: 9227864]

4. Nouwen JL, van Belkum A, Verbrugh HA. Determinants of Staphylococcus aureus nasal carriage. Neth J Med. 2001; 59(3):126-33. [PubMed: 11583828]

5. Nouwen JL, et al. Predicting the Staphylococcus aureus nasal carrier state: derivation and validation of a "culture rule". Clin Infect Dis. 2004; 39(6):806-11. [PubMed: 15472812]

6. Ellis MW, et al. Natural history of community-acquired methicillin-resistant Staphylococcus aureus colonization and infection in soldiers. Clin Infect Dis. 2004; 39(7):971-9. [PubMed: 15472848]

7. Wertheim HF, et al. The role of nasal carriage in Staphylococcus aureus infections. Lancet Infect Dis. 2005; 5(12):751-62. [PubMed: 16310147]

8. Faden $\mathrm{H}$, et al. Importance of colonization site in the current epidemic of staphylococcal skin abscesses. Pediatrics. 2010; 125(3):e618-24. [PubMed: 20156893]

9. Methicillin-resistant Staphylococcus aureus infections in correctional facilities---Georgia, California, and Texas, 2001-2003. MMWR Morbidity and mortality weekly report. 2003; 52(41): 992-6. [PubMed: 14561958] 
10. Pan ES, et al. Increasing prevalence of methicillin-resistant Staphylococcus aureus infection in California jails. Clinical infectious diseases : an official publication of the Infectious Diseases Society of America. 2003; 37(10):1384-8. [PubMed: 14583874]

11. Salgado CD, Farr BM, Calfee DP. Community-acquired methicillin-resistant Staphylococcus aureus: a meta-analysis of prevalence and risk factors. Clin Infect Dis. 2003; 36(2):131-9. [PubMed: 12522744]

12. Kazakova SV, et al. A clone of methicillin-resistant Staphylococcus aureus among professional football players. N Engl J Med. 2005; 352(5):468-75. [PubMed: 15689585]

13. Weber JT. Community-associated methicillin-resistant Staphylococcus aureus. Clin Infect Dis. 2005; 41(4):S269-72. [PubMed: 16032563]

14. Kirkland EB, Adams BB. Methicillin-resistant Staphylococcus aureus and athletes. J Am Acad Dermatol. 2008; 59(3):494-502. [PubMed: 18550208]

15. Cohen PR. The skin in the gym: a comprehensive review of the cutaneous manifestations of community-acquired methicillin-resistant Staphylococcus aureus infection in athletes. Clin Dermatol. 2008; 26(1):16-26. [PubMed: 18280900]

16. Nouwen J, et al. Human factor in Staphylococcus aureus nasal carriage. Infection and immunity. 2004; 72(11):6685-8. [PubMed: 15501803]

17. van Belkum A, et al. Reclassification of Staphylococcus aureus nasal carriage types. J Infect Dis. 2009; 199(12):1820-6. [PubMed: 19419332]

18. Rice SG. Medical conditions affecting sports participation. Pediatrics. 2008; 121(4):841-8. [PubMed: 18381550]

19. Creech CB, et al. One-year surveillance of methicillin-resistant Staphylococcus aureus nasal colonization and skin and soft tissue infections in collegiate athletes. Arch Pediatr Adolesc Med. 2010; 164(7):615-20. [PubMed: 20603460]

20. Jimenez-Truque N, et al. Longitudinal Assessment of Colonization With Staphylococcus aureus in Healthy Collegiate Athletes. J Pediatric Infect Dis Soc. 2016; 5(2):105-13. [PubMed: 27199467]

21. Creech CB 2nd, et al. Increasing rates of nasal carriage of methicillin-resistant Staphylococcus aureus in healthy children. Pediatr Infect Dis J. 2005; 24(7):617-21. [PubMed: 15999003]

22. Oliveira DC, de Lencastre H. Multiplex PCR strategy for rapid identification of structural types and variants of the mec element in methicillin-resistant Staphylococcus aureus. Antimicrob Agents Chemother. 2002; 46(7):2155-61. [PubMed: 12069968]

23. Lina G, et al. Involvement of Panton-Valentine leukocidin-producing Staphylococcus aureus in primary skin infections and pneumonia. Clin Infect Dis. 1999; 29(5):1128-32. [PubMed: 10524952]

24. Kondo Y, et al. Combination of multiplex PCRs for staphylococcal cassette chromosome mec type assignment: rapid identification system for mec, ccr, and major differences in junkyard regions. Antimicrob Agents Chemother. 2007; 51(1):264-74. [PubMed: 17043114]

25. Harris PA, et al. Research electronic data capture (REDCap)--a metadata-driven methodology and workflow process for providing translational research informatics support. Journal of biomedical informatics. 2009; 42(2):377-81. [PubMed: 18929686]

26. Eriksen NH, et al. Carriage of Staphylococcus aureus among 104 healthy persons during a 19month period. Epidemiology and infection. 1995; 115(1):51-60. [PubMed: 7641838]

27. Harrell, FE. Springer series in statistics. New York: Springer; 2001. Regression Modeling Strategies: With Applications to Linear Models, Logistic Regression, and Survival Analysis; p. 568

28. Grosset-Janin A, Nicolas X, Saraux A. Sport and infectious risk: a systematic review of the literature over 20 years. Med Mal Infect. 2012; 42(11):533-44. [PubMed: 23116704]

29. Cole AM, et al. Determinants of Staphylococcus aureus nasal carriage. Clinical and diagnostic laboratory immunology. 2001; 8(6):1064-9. [PubMed: 11687441]

30. Graham PL 3rd, Lin SX, Larson EL. A U.S. population-based survey of Staphylococcus aureus colonization. Ann Intern Med. 2006; 144(5):318-25. [PubMed: 16520472]

31. Lebon A, et al. Dynamics and determinants of Staphylococcus aureus carriage in infancy: the Generation R Study. J Clin Microbiol. 2008; 46(10):3517-21. [PubMed: 18667593] 
32. Kuehnert MJ, et al. Prevalence of Staphylococcus aureus nasal colonization in the United States, 2001-2002. The Journal of infectious diseases. 2006; 193(2):172-9. [PubMed: 16362880]

33. Williams RE. Healthy carriage of Staphylococcus aureus: its prevalence and importance. Bacteriological reviews. 1963; 27:56-71. [PubMed: 14000926]

\section{List of Abbreviation}

MRSA methicillin-resistant Staphylococcus aureus

CA-MRSA community-associated methicillin-resistant Staphylococcus aureus

MSSA methicillin-sensitive Staphylococcus aureus

PVL Panton-Valentine Leukocidin

SCCmec Staphylococcal Cassette Chromosome mec 


\section{Key Points}

$S$. aureus carriage was common in contact sports athletes, particularly in the football team.

Athletes who played contact sports were more likely to carry MRSA over time and tended to carry $S$. aureus for longer periods of time than athletes in non-contact sports.

Athletes engaged in contacts sports also acquired $S$. aureus more quickly than their non-contact sports counterparts. 


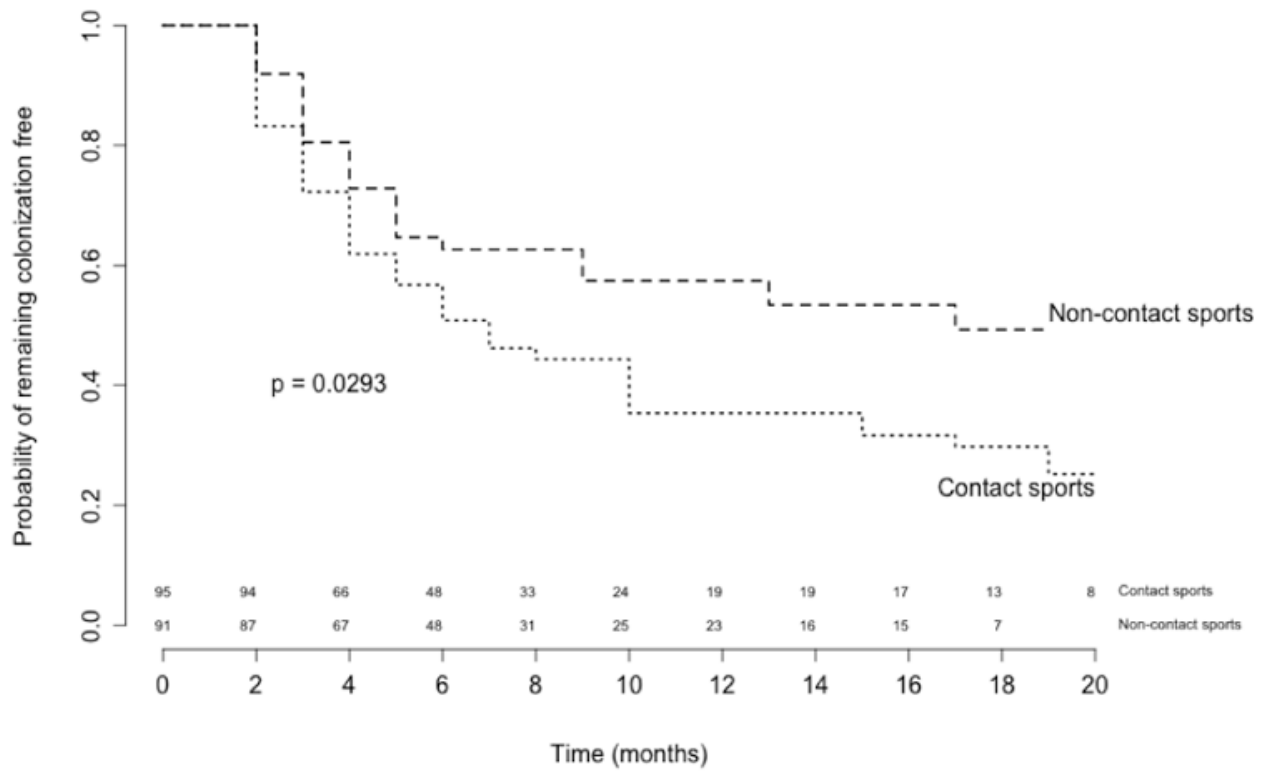

Figure 1. Kaplan-Meier curve for the time to becoming colonized with $S$. aureus in a cohort of 186 college athletes, by type of sport 


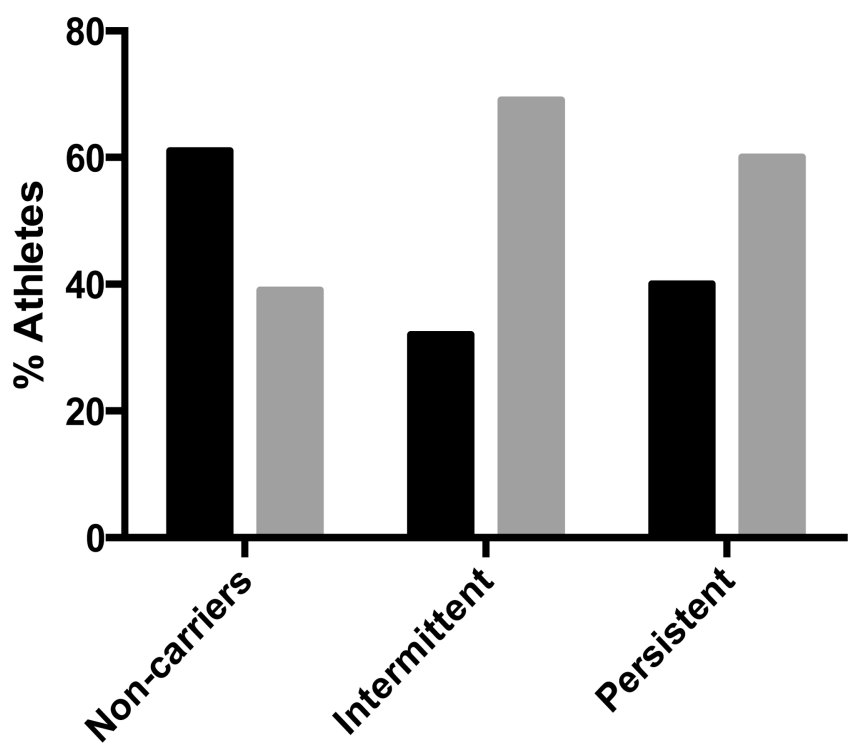

Non-contact

Colonization Profile

Figure 2.

Percentage of athletes who were never, intermittently, or persistently colonized with $S$. aureus by type of sport. Those who were staphylococcal carriers during $\geq 80 \%$ of the times at which they are sampled were classified as persistent carriers; those who were never colonized were non-carriers, and those colonized at least once, but $<80 \%$ of the times at which they were swabbed, were classified as intermittent carriers. 
Table 1

Demographics and medical history at enrollment for a cohort of 377 college student athletes at Vanderbilt University, by type of sport

\begin{tabular}{|c|c|c|c|c|}
\hline Variable & $\begin{array}{l}\text { Total Cohort } \\
\mathbf{N}=377 \text { N(\%) }\end{array}$ & $\begin{array}{l}\text { Non-contact } \\
\mathbf{N}=153 \mathbf{N}(\%)\end{array}$ & $\begin{array}{r}\text { Contact } \\
\mathrm{N}=224 \mathrm{~N}(\%)\end{array}$ & $\begin{array}{r}\text { Time-to-event cohort } \\
\mathrm{N}=186 \mathrm{~N}(\%)\end{array}$ \\
\hline \multicolumn{5}{|l|}{ Gender } \\
\hline Female & $161(42.71)$ & $79(51.63)$ & $82(36.61)$ & $94(50.54)$ \\
\hline Male & $216(57.29)$ & $74(48.37)$ & $142(63.39)$ & $92(49.46)$ \\
\hline \multicolumn{5}{|l|}{ Race/Ethnicity } \\
\hline Caucasian & $280(74.27)$ & $133(86.93)$ & $147(65.62)$ & $141(75.81)$ \\
\hline African-American & $80(21.22)$ & $11(7.19)$ & $69(30.80)$ & $38(20.43)$ \\
\hline Other & $17(4.51)$ & $9(5.88)$ & $8(3.57)$ & $7(3.76)$ \\
\hline \multicolumn{5}{|l|}{ College Year } \\
\hline Freshman & $149(39.52)$ & $64(41.83)$ & $85(37.95)$ & $79(42.47)$ \\
\hline Sophomore & $87(23.08)$ & $36(23.53)$ & $51(22.77)$ & $41(22.04)$ \\
\hline Junior & $70(18.57)$ & $23(15.03)$ & $47(20.98)$ & $34(18.28)$ \\
\hline Senior & $59(15.65)$ & 18 (11.76) & $41(18.30)$ & $25(13.44)$ \\
\hline Trainers & $12(3.18)$ & $12(7.84)$ & $0(0.00)$ & $7(3.76)$ \\
\hline \multicolumn{5}{|c|}{ History of $S$. aureus infection } \\
\hline No & $355(94.16)$ & $148(96.73)$ & $207(92.41)$ & $179(96.24)$ \\
\hline Yes & $22(5.84)$ & $5(3.27)$ & $17(7.59)$ & $7(3.76)$ \\
\hline \multicolumn{5}{|c|}{ S. aureus infection in contacts } \\
\hline No & $261(69.23)$ & $122(79.74)$ & $139(62.05)$ & $127(68.28)$ \\
\hline Yes & $116(30.77)$ & $31(20.26)$ & $85(37.95)$ & $59(31.72)$ \\
\hline \multicolumn{5}{|l|}{ Previous surgeries } \\
\hline No & $275(72.94)$ & $126(82.35)$ & $149(66.52)$ & $140(75.27)$ \\
\hline Yes & $102(27.06)$ & $27(17.65)$ & $75(33.48)$ & $46(24.73)$ \\
\hline \multicolumn{5}{|l|}{ Comorbidities } \\
\hline No & $362(96.02)$ & $148(96.73)$ & $214(95.54)$ & $180(96.77)$ \\
\hline Yes & $15(3.98)$ & $5(3.27)$ & $10(4.46)$ & $6(3.23)$ \\
\hline \multicolumn{5}{|c|}{ Antibiotics in Previous 6 Months } \\
\hline No & $329(87.27)$ & $132(86.27)$ & 197 (87.95) & $156(83.87)$ \\
\hline Yes & $48(12.73)$ & $21(13.73)$ & $27(12.05)$ & $30(16.13)$ \\
\hline \multicolumn{5}{|c|}{ Staphylococcal carrier profile ${ }^{a}$} \\
\hline Non-carriers & $90(23.87)$ & $55(35.95)$ & $35(15.62)$ & $90(48.39)$ \\
\hline Intermittent carriers & $200(53.05)$ & $63(41.18)$ & $137(61.16)$ & $96(51.61)$ \\
\hline Persistent carriers & 87 (23.08) & $35(22.88)$ & $52(23.21)$ & $0(0.00)$ \\
\hline \multicolumn{5}{|c|}{ Number of months swabbed } \\
\hline 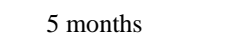 & $112(29.71)$ & $80(52.29)$ & $32(14.28)$ & $64(34.41)$ \\
\hline$<5$ and $<13$ months & $162(42.97)$ & $60(39.21)$ & $102(45.54)$ & $76(40.86)$ \\
\hline$\geq 13$ months & $103(27.32)$ & $13(8.50)$ & $90(40.18)$ & $46(24.73)$ \\
\hline
\end{tabular}

${ }^{a}$ Staphylococcal carrier profile was measured over up to 18 months 


\section{롤 \\ 일}

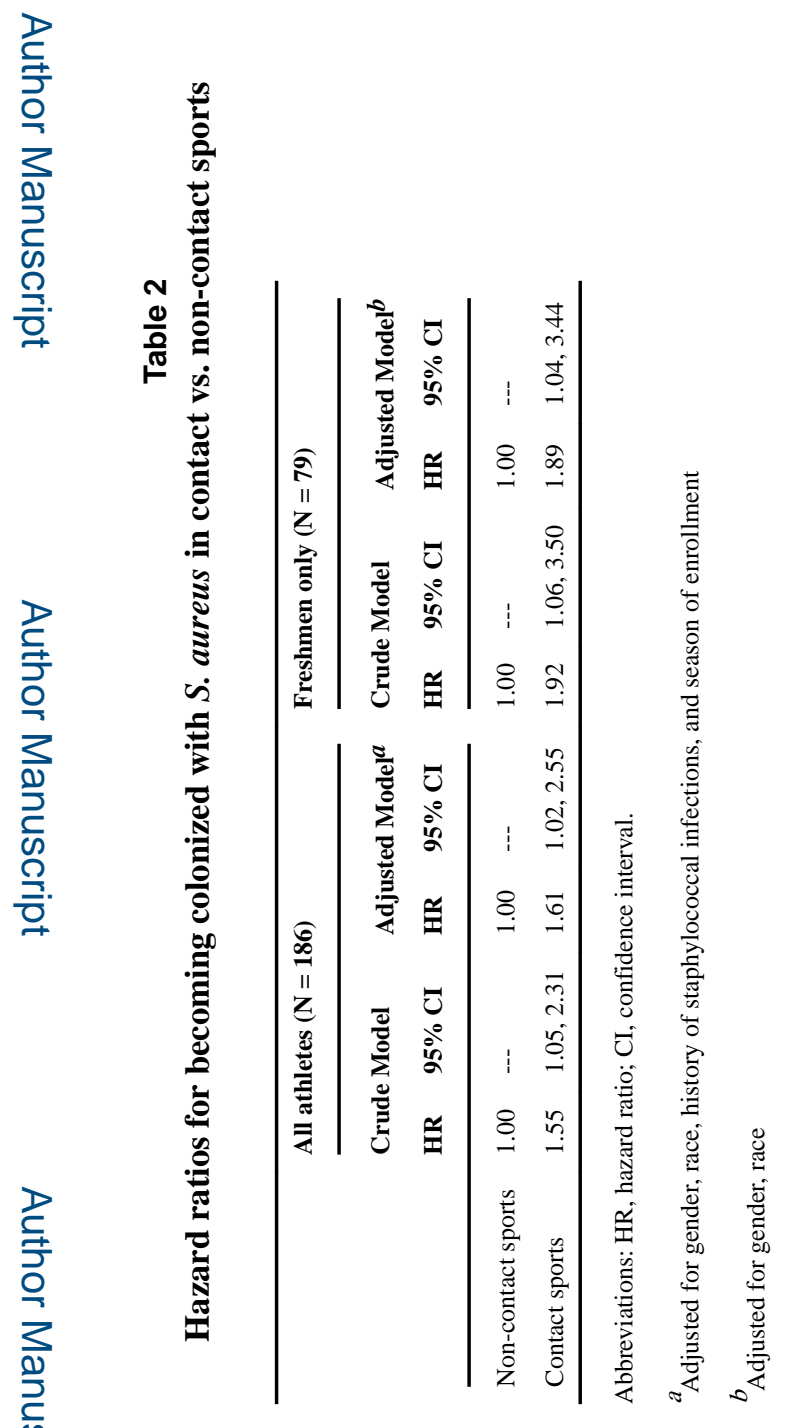

Sports Med. Author manuscript; available in PMC 2018 May 01. 
Table 3

Odds ratios comparing colonization with MRSA or MSSA vs. no colonization over time for a cohort of 377 college student athletes

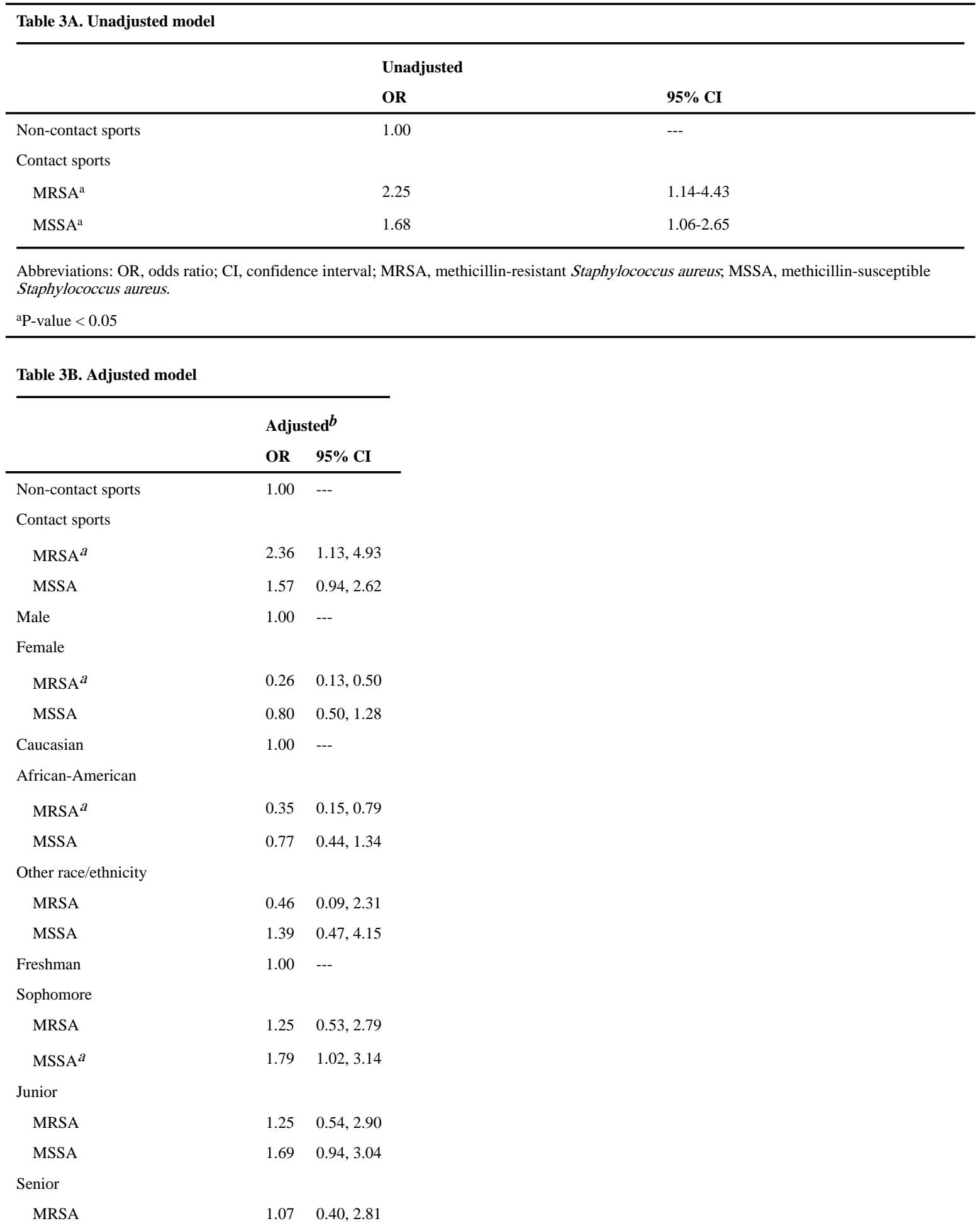


Table 3B. Adjusted model

\begin{tabular}{|c|c|c|}
\hline & \multicolumn{2}{|c|}{ Adjusted $b$} \\
\hline & OR & $95 \% \mathrm{CI}$ \\
\hline MSSA & 1.20 & $0.61,2.37$ \\
\hline \multicolumn{3}{|l|}{ Trainers } \\
\hline MRSA & 0.97 & $0.14,6.69$ \\
\hline MSSA & 0.82 & $0.21,3.27$ \\
\hline No history of $S$. aureus infection & 1.00 & --- \\
\hline \multicolumn{3}{|l|}{ History of $S$. aureus infection } \\
\hline MRSA $^{a}$ & 5.35 & $1.51,18.87$ \\
\hline MSSA & 2.19 & $0.86,5.52$ \\
\hline No $S$. aureus infections in contacts & 1.00 & --- \\
\hline \multicolumn{3}{|l|}{ Staphylococcal infections in contacts } \\
\hline MRSA $^{a}$ & 0.45 & $0.22,0.94$ \\
\hline MSSA & 0.72 & $0.43,1.20$ \\
\hline No comorbidities & 1.00 & --- \\
\hline \multicolumn{3}{|l|}{ Any comorbidities } \\
\hline MRSA & 2.90 & $0.65,13.01$ \\
\hline MSSA & 1.71 & $0.59,5.01$ \\
\hline No previous surgeries & 1.00 & --- \\
\hline \multicolumn{3}{|l|}{ Any Previous surgeries } \\
\hline MRSA & 1.28 & $0.63,2.60$ \\
\hline MSSA & 1.31 & $0.80,2.15$ \\
\hline No previous antibiotics & 1.00 & --- \\
\hline \multicolumn{3}{|l|}{ Previous antibiotics } \\
\hline MRSA & 1.29 & $0.50,3.34$ \\
\hline MSSA & 0.59 & $0.30,1.18$ \\
\hline
\end{tabular}

Abbreviations: OR, odds ratio; CI, confidence interval; MRSA, methicillin-resistant Staphylococcus aureus; MSSA, methicillin-susceptible Staphylococcus aureus.

${ }^{a}$ P-value $<0.05$

${ }^{b}$ Adjusted for gender, race (African-American, Caucasian, other), year (Freshman, Junior, Sophomore, Senior, trainer), history of staphylococcal infections, staphylococcal infections in contacts, any comorbidities, previous surgeries, previous antibiotics, contact sport - gender interaction, and time 